Document downloaded from:

http://hdl.handle.net/10251/99458

This paper must be cited as:

J. Antonino-Daviu; Riera-Guasp, M.; Manuel Pineda-Sanchez; Pérez, RB. (2009). A critical comparison between DWT and Hilbert-Huang-based methods for the diagnosis of rotor bar failures in induction machines. IEEE Transactions on Industry Applications. 45(5):17941803. doi:10.1109/TIA.2009.2027558

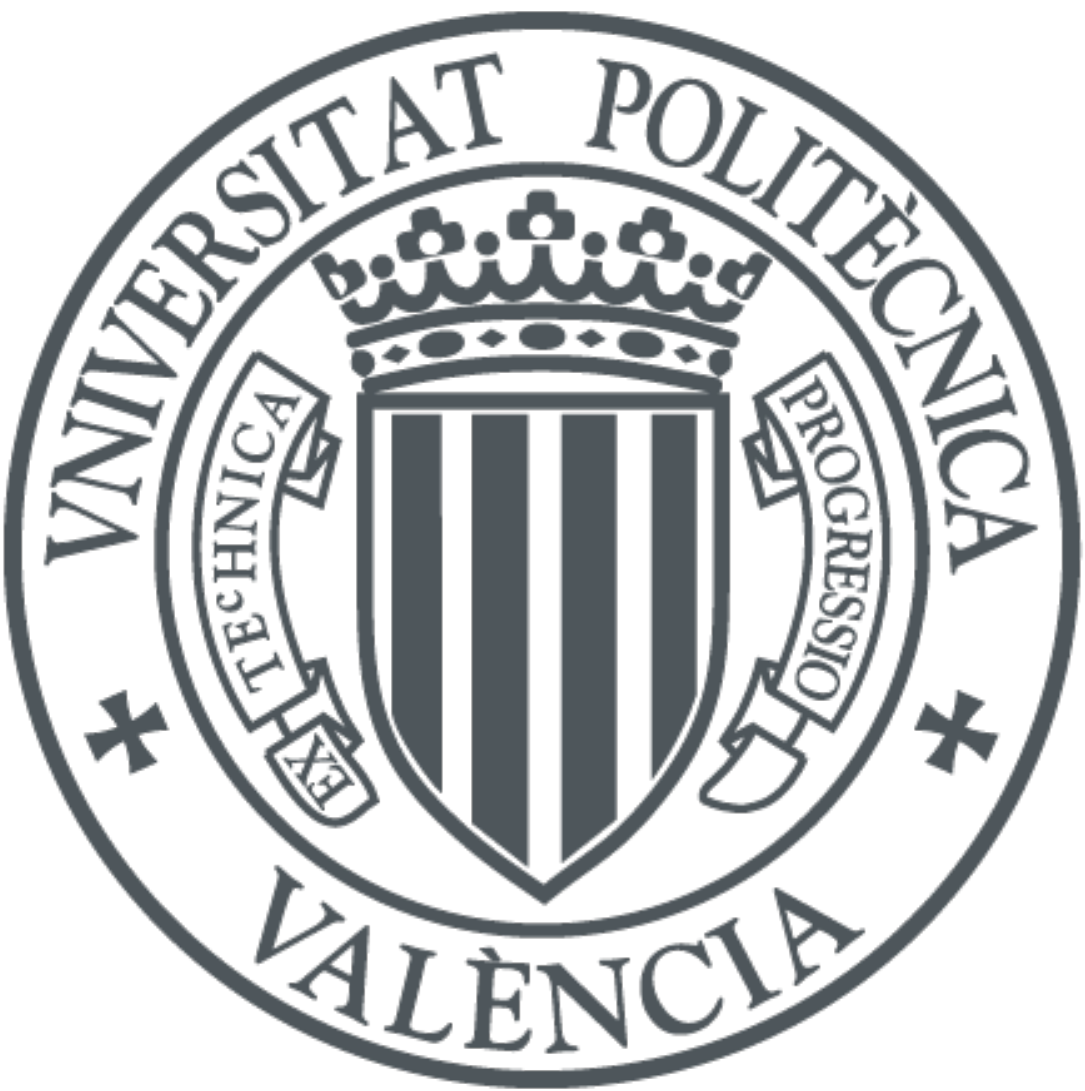

The final publication is available at

http://doi.org/10.1109/TIA.2009.2027558

Copyright Institute of Electrical and Electronics Engineers

Additional Information 


\section{A critical comparison between DWT and Hilbert- Huang-based methods for the diagnosis of rotor bar failures in induction machines}

\author{
J.A. Antonino-Daviu, M.Riera-Guasp , M. Pineda-Sanchez \\ Depto. Ingenieria Electrica \\ Instituto de Ingeniería Energetica \\ Universidad Politecnica de Valencia \\ Valencia, SPAIN \\ joanda@die.upv.es
}

\begin{abstract}
In this paper, a cutting edge time frequency decomposition tool, the Hilbert-Huang Transform (HHT) is applied to the stator startup current in order to diagnose the presence of rotor asymmetries in induction machines. The objective is to extract the evolution during the startup transient of the left sideband harmonic (LSH) caused by the asymmetry, which constitutes a reliable evidence of the presence of the fault. The validity of the diagnosis methodology is assessed through several tests developed using real experimental signals. Moreover, in the paper, an analytical comparison with an alternative time-frequency decomposition tool, the Discrete Wavelet Transform (DWT) is carried out. This tool was applied in previous works to the transient extraction of fault-related components, with satisfactory results, even in cases in which the classical Fourier approach does not lead to correct results. The results of the application of the HHT and DWT are analyzed and compared, obtaining novel conclusions about their respective suitability for the transient extraction of asymmetry-related components, as well as the equivalence, with regards to the LSH extraction, between their basic components: intrinsic mode function, for the HHT, and approximation signal for the DWT.
\end{abstract}

Keywords- rotor asymmetries; startup transient; fault diagnosis; Hilbert-Huang Transform; wavelet analysis;

\section{INTRODUCTION}

During these recent years, predictive maintenance of electrical machinery has drawn the attention of many companies, due to the huge costs caused by unexpected failures in these machines. Early detection of broken rotor bars in induction machines has become a field of special interest. This constitutes a dangerous fault, which might not show apparent symptoms of its presence during its early stages, propagating towards the adjacent bars and leading to an abrupt collapse of the machine unless continuous monitoring of certain quantities has been carried out $[1,2]$.

The most spread method for the diagnosis of rotor asymmetries in the industrial environment is based on the FFT analysis of the steady-state stator current and the study of two harmonic components appearing around the fundamental component (sideband components) [3-6]. This approach provides robust results when the machine operates under a certain level of load and perfect stationary regime, but it has some important drawbacks when it is applied to diagnose the condition of unloaded or light-loaded machines, machines

\author{
R. B. Pérez \\ Nuclear Engineering Department \\ University of Tennessee \\ Knoxville, Tennessee, USA \\ rperez1@utk.edu
}

driving variable torque loads (for instance, motors driving mills, compressors, gear reducers...) or machines supplied with fluctuating voltages [4, 6-7]. Some methods based on the study of the transient processes of the machine [8] and, more concretely, of the startup transient [7, 9-15], have been developed recently. The implicit or explicit basis of these techniques lies in the detection of the left sideband harmonic (LSH), associated with the fault, during that transient. The characteristic evolution of this component constitutes a reliable evidence of the presence of the breakage. A method based on the application of the Discrete Wavelet Transform (DWT) to the stator startup current was introduced recently by the authors [7, 12-14]. Unlike other approaches, the method is focused on the study of the high level wavelet signals (approximation signal and detail signals) resulting from the analysis. The use of the wavelet signals (instead of the coefficients) enables a clearer interpretation of the physical phenomenon taking place in the machine; clear patterns caused by the evolution of asymmetry-related components appear in these signals. The patterns make possible the diagnosis of the fault, even in cases in which the Fourier analysis does not lead to correct diagnostics [7].

Despite the satisfactory results of the DWT method for a wide range of tested motors (from $1.1 \mathrm{~kW}$ up to several MW), some issues still remain. Firstly, despite not being a critical problem, the selection of the mother wavelet for the application of the DWT is somehow arbitrary, since there is not a clear rule for selecting the optimal mother wavelet for a specific application. With regard to this fact, design of specific wavelets, suitable for the extraction of the LSH, has been proposed in recent works [15]. Other issue lies in the overlap between frequency bands associated with the wavelet signals according to Mallat algorithm, which could make not suitable the selection of a particular mother wavelet, mainly if its order is low (low number of filter coefficients) $[13,25]$. Also, the boundary distortion introduced by the transform might, in some specific cases, make difficult the identification of the left sideband component evolution, mainly if the startup transient is very fast.

Recently, a novel time-frequency decomposition tool, the Hilbert-Huang Transform (HHT), has been proposed. Its application has been mainly focused in the predictive maintenance of Nuclear Reactors as well as applications in other scientific areas [16]. Some recent contributions dealing 
with diagnosis of bearing faults in electrical machinery have been also presented [17-18], although they deal with the study of other quantities and regimes. This tool has been claimed to provide certain advantages in comparison with wavelet-based and other time-frequency decomposition tools.

In any case, it has to be remarked that the methodology here developed, is valid for machines started direct on-line. Further modifications would be needed for adapting the philosophy to inverted-fed machines [30].

The main objective of this paper is to analyse the viability of the HHT when diagnosing the presence of rotor asymmetries in an induction machine. The study will be focused in its performance regarding the transient detection and extraction of the LSH, drawing the attention on some aspects which might be improved with the HHT with respect to DWT (no necessity of selection of mother wavelet, avoidance of the shift-variance problem caused by the decimation inherent to the DWT [1920], or possibility of application of automatic image recognition techniques). Results from the tests carried out on real machines are presented. Finally, advantages and drawbacks of each approach are discussed. Important conclusions are reached about the equivalence between their basic decomposition components, regarding the transient extraction of the LSH; the intrinsic mode function (IMF) and the approximation signal $\left(a_{n}\right)$, covering frequencies below the fundamental.

\section{PHENOMENON DESCRIPTION}

When a rotor bar breaks, two harmonics (sideband components) appear around the supply frequency in the FFT spectrum of the steady-state current. Their frequencies are given by (1) ( $s=$ slip, $f=$ supply frequency) [3].

$$
f_{S H}=f \cdot(1 \pm 2 \cdot s)
$$

During the startup, the left sideband harmonic (LSH) (negative sign in (1)), evolves in a particular way in frequency and amplitude. In [12] the theoretical waveform of this harmonic during that transient was justified using the space vector theory and the approach introduced by Deleroi [21] for studying the bar breakage physical phenomenon. This waveform, calculated for a $1.1 \mathrm{~kW}$ commercial cage motor with a broken bar is shown in Fig. 1 .

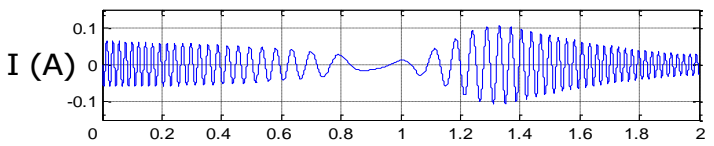

Figure 1. Evolution of the LSH in a stator phase during the startup transient.

The evolution of the frequency and amplitude of the LSH during the transient is described next:

- Its frequency $\left(f_{L S H}\right)$, at the beginning, is equal to the supply frequency $f$. As the slip $s$ decreases from 1 to almost 0 during the startup, the frequency of the LSH decreases firstly until becoming zero. Then, it increases until reaching a value close to the supply frequency $f$. This characteristic evolution in frequency is according to the expression (1) (component with negative sign), valid for steady-state.
- Its amplitude decreases firstly from an initial value, until the slip equals 0.5 . Then, it increases until reaching a maximum value (higher than the initial amplitude) and it decreases again until reaching the steady-state amplitude. The description and physical interpretation of this evolution is also carried out in [12].

\section{HILBERT-HUANG TRANSFORM AND DISCRETE WAVELET TRANSFORM}

\section{A. Hilbert-Huang Transform (HHT)}

Recently, Huang and coworkers [16, 22] have introduced a new signal analysis technique based on the decomposition of a complex signal onto a sum of quasi-monocomponent ones (Intrinsic Mode Functions, IMF's) by using an empirical approach (Empiric Mode Decomposition, EMD), and on their representation within the context of the Complex Trace Method first introduced by Gabor [23]. This method, formulates a signal, $X(q)$, $(q$ representing either time or an spatial coordinate) as the real part of a complex trace, $\mathrm{Z}(q)$,

$$
Z(q)=X(q)+i Y(q)
$$

where the imaginary part, $Y(q)$, is the Hilbert transform:

$$
Y(q)=(1 / \pi) \cdot P V \cdot \int_{-\infty}^{\infty} X\left(q^{\prime}\right) d q^{\prime} /\left(q-q^{\prime}\right)
$$

where $P V$ indicates the principal value of the singular integral. The complex conjugate pair $(X(q) ; Y(q))$ defines the amplitude, $a(q)$, and phase $\theta(q)$, as an analytical function of the $q$-variable:

$$
Z(q)=a(q) \cdot e^{i \theta(q)}
$$

where,

$a(q)=\left[X^{2}(q)+Y^{2}(q)\right]^{1 / 2} \quad, \quad \tan \theta(q)=(Y(q) / X(q))$

with the instantaneous frequency defined as:

$$
\omega(q)=\mathrm{d} \theta(q) / \mathrm{d} q
$$

The Complex Trace formalism then defines the concepts of instantaneous amplitude, phase and frequency such that the original signal can be expressed in terms of a Fourier-like expansion based on these concepts [16].

The aforementioned process as well as the definition of instantaneous frequency work well for mono-component signals. However, for many real applications the signals are multi-component and often noise corrupted. In these cases, the Complex Trace concept fails because the Hilbert transform processing of those noisy waves generates spurious amplitudes at negative frequencies. Huang [16, 22] developed an entirely new approach to signal analysis to avoid generating unphysical results. To this end, the Hilbert transform is not directly applied to the signal itself but to each of the members of an empirical decomposition of the signal into intrinsic mode functions (IMF's). These IMF's are individual, nearly monocomponent signals with 'Hilbert-friendly' waveforms, to 
which the instantaneous frequency defined by (6) can be applied [24].

The algorithm to create the IMF's, referred to as "sifting", is both elegant and simple. First, the local extrema of the data are identified and used to create upper and lower envelopes which enclose the signal completely. From this envelope a running mean is created. By subtracting this "mean" from the data one obtains a new function, which must have the same number of zero crossings and extrema (i.e. it exhibits symmetry across the $q$-axis). If the function so constructed does not satisfy this criterion, the "sifting" process continues until some acceptable tolerance is reached [22]. The resulting $q$-series is the first 'IMF', $c_{1}(q)$, and contains the highest frequency oscillations found in the data (the shortest time scales). The IMF1, is then subtracted from the original data, and this difference $R l$ is taken as if it were the original signal and then the sifting process is applied to the new signal.

The process of finding modes, $c_{j}(q)$, continues until the last mode, the residue $R n$, is found which will contain the trend (i.e. , the "time -varying" mean). Thus, the signal, $X(q)$, is given by the sum:

$$
X(q)=\sum_{j=1}^{n} c_{j}(q)+R n
$$

The issues of completeness and orthogonality of the IMFexpansion are discussed by Huang in [22].

Once the IMF's are obtained, the Hilbert transform can be applied to each individual IMF, computing the instantaneous frequency and amplitude using (5) and (6). After applying the Hilbert transform to each IMF, the signal can be expressed according to (8), where $a_{j}(q)$ and $w_{j}(q)$ are, respectively, the instantaneous amplitude and frequency corresponding to each $\operatorname{IMF} c_{j}(q)$.

$$
X(q)=\operatorname{Re}\left(\sum_{j=1}^{n} a_{j}(q) \cdot e^{i \int \omega_{j}(q) \cdot d q}\right)
$$

This expression enables the representation of the instantaneous amplitude and frequency as functions of $q$ in a three-dimensional plot or contour map. The time-frequency representation of the amplitude is named Hilbert-Huang spectrum, $\mathrm{H}(w, q)[24]$.

After defining the Hilbert-Huang (HH) spectrum, the marginal spectrum can be also defined according to (9), where $Q$ is the total data length [17];

$$
h(w)=\int_{0}^{Q} H(w, q) \cdot d q
$$

Whereas the $\mathrm{HH}$ spectrum offers a measure of the amplitude contribution for each frequency and time, the marginal spectrum (power spectral density) offers a measure of the total amplitude (or energy) contribution from each frequency [17]. The frequency in the marginal spectrum indicates only the likelihood that an oscillation with such a frequency exists; the exact occurrence time of that oscillation is given in the full Hilbert spectrum [22].

\section{B. Discrete Wavelet Transform (DWT)}

The DWT decomposes the sampled signal being analysed, $y\left(y_{1}, y_{2}, \ldots y_{\mathrm{N}}\right)$, onto an approximation signal $a_{\mathrm{n}}$ and several detail signals $d_{\mathrm{j}}[7,20]$, according to (10).

$$
y(t)=\sum_{i} \alpha_{i}^{n} \cdot \varphi_{i}^{n}(t)+\sum_{j=1}^{n} \sum_{i} \beta_{i}^{j} \cdot \psi_{i}^{j}(t)=a_{n}+d_{n}+\ldots+d_{1}
$$

$\alpha_{i}^{n}, \beta_{i}^{j}$ are the scaling and wavelet coefficients, $\varphi^{n}(\mathbf{t})$ is the scaling function at level $n$ and $\psi^{j}(\mathbf{t})$ the wavelet function at level $j ; n$ is the decomposition level; $a_{n}$ is the approximation signal at level $n$ and $d_{j}$ the detail signal at level $j$ [20].

Mallat algorithm shows that each wavelet signal is associated with a certain frequency band. If $f_{\mathrm{s}}$ (samples/s) is the sampling rate used for capturing $y$, then the detail signal $d_{\mathrm{j}}$ contains the information concerning the signal components with frequencies included in the interval $\left[2^{-(\mathrm{j}+1)} \cdot f_{s}, 2^{-\mathrm{j}} \cdot f_{s}\right] \mathrm{Hz}$. The approximation signal $a_{\mathrm{n}}$ includes the low frequency components of the signal, belonging to the interval $\left[0,2^{-(\mathrm{n}+1)} \cdot f_{s}\right]$ $\mathrm{Hz}$ [7]. DWT performs then the filtering process shown in Fig. 2. As observed, the filtering is not ideal, a fact leading to a certain overlap between adjacent frequency bands [25]. This can be problematic, since some frequency components (specially the fundamental component) can be partially filtered in the adjacent band, masking the components within that band.

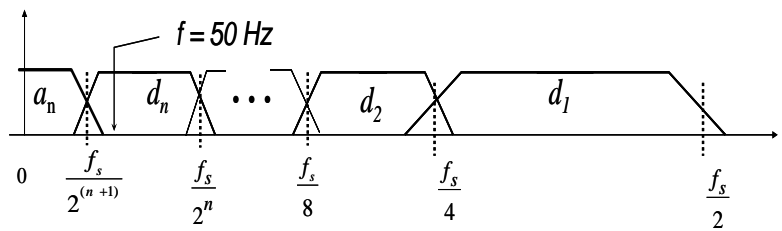

Figure 2. Filtering process performed by the DWT.

\section{EXPERIMENTAL RESULTS}

Tests were developed on $1.1 \mathrm{~kW}$ cage induction motor. The motor was directly coupled to a $10 \mathrm{~kW}$ DC machine acting as a load. Interchangeable rotors with different number of breakages were considered. The breakages were forced in the laboratory, drilling the holes in the selected bars.

\section{A. Application of the HHT}

Six transient data sets of induction machines stator current subject to different number of bar breakages and load conditions were analyzed (Table I). Each data set was decomposed into two intrinsic modes (IMF1 and IMF2) and their respective $\mathrm{HH}$ spectra calculated. The second intrinsic modes for the six data sets are shown in Fig. 3. In Figs. 4 to 9 the input signal (a), speed during the startup (b); HH spectrum of the first intrinsic mode IMF1 (c), HH spectrum of the second intrinsic mode IMF2 (d) and the power spectral density of the input signal (e) are plotted for each one of the data sets.

Inspection of Fig. 3 reveals that the IMF2 remains low if the machine is healthy ( $\mathrm{q} 1$ and $\mathrm{q} 4)$ whereas it increases its 
amplitude for the faulty machine. Indeed, the amplitude of IMF2 increases with the number of broken bars (compare q2$\mathrm{q} 3$ and q5-q6). A deeper analysis reveals that the waveform of the IMF2 for the faulty machine is quite similar to the theoretical evolution of the left sideband component shown in Fig. 1 and justified in [12]. This fact indicates that the IMF2 reflects the time evolution of the frequency components below $50 \mathrm{~Hz}$ (supply frequency) and, therefore, IMF2 is extracting the evolution of the left sideband (LSH) during the startup transient, since it is the unique significant component within that range of frequencies.

It has to be remarked that the IMF's are obtained from an empiric methodology (EMD), and so, there are no analytic equations enabling the deduction of the range of frequencies of the components included in a specific IMF. So, the interpretation of the IMF's has to be based on physical reasoning, as exposed in the precedent and next paragraphs.

A deeper analysis of Figs. 4 to 9, leads to additional interesting conclusions:

- Firstly, as a result of the HHT analysis of each data set, an IMF1 is obtained. Figs. 4 to 9, sections (c), show the IMF1 spectrum obtained for the six analyzed cases. In all these cases a single component at $50 \mathrm{~Hz}$ is detected along the whole length of the startup. Moreover, the HH spectrum of each IMF1 shows that the amplitude of this component decreases as the current decreases during the startup (from red color to blue color). These facts indicate that the IMF1 extracts the main component at the supply frequency from the tested signal.

- As commented above, the second intrinsic mode function (IMF2) resulting from the analysis of each data set reflects the evolution of the rest of frequency components below $50 \mathrm{~Hz}$. This is confirmed when analyzing the $\mathrm{HH}$ spectrum for each IMF2; for the healthy machine (Fig.4 (d) and 7 (d)) only slight traces appear in the $\mathrm{HH}$ spectra due to the fact that no sideband component exist in the machine, so no significant components exist within the frequency band reflected by IMF2. Nevertheless, for the faulty machine (Figs. 5 (d), 6 (d), 8(d) and 9(d)) some components are detected in the IMF2 spectra. The pattern appearing in those spectra is quite characteristic; as the startup progresses, there is a first stretch in which the frequency decreases from $50 \mathrm{~Hz}$ to $0 \mathrm{~Hz}$ and a second one in which the frequency increases again up to $50 \mathrm{~Hz}$. In addition, the color of the spectra informs about the amplitude of the LSH at each time; a higher amplitude is detected during the second stretch. This characteristic pattern found in the HHT current spectra of the faulty machines fits perfectly the theoretical waveform of the LSH shown in Fig. 1, confirming again that the HHT (and the EMD) is a suitable approach for diagnosing rotor asymmetries, since it enables a clear extraction and detection of the LSH produced by the fault. As it will be seen, the pattern described in the HHT spectrum keeps a certain similitude with the characteristic pattern obtained with the second approach based on the DWT method (Fig. 13), also caused by the transient evolution in frequency of the LSH.

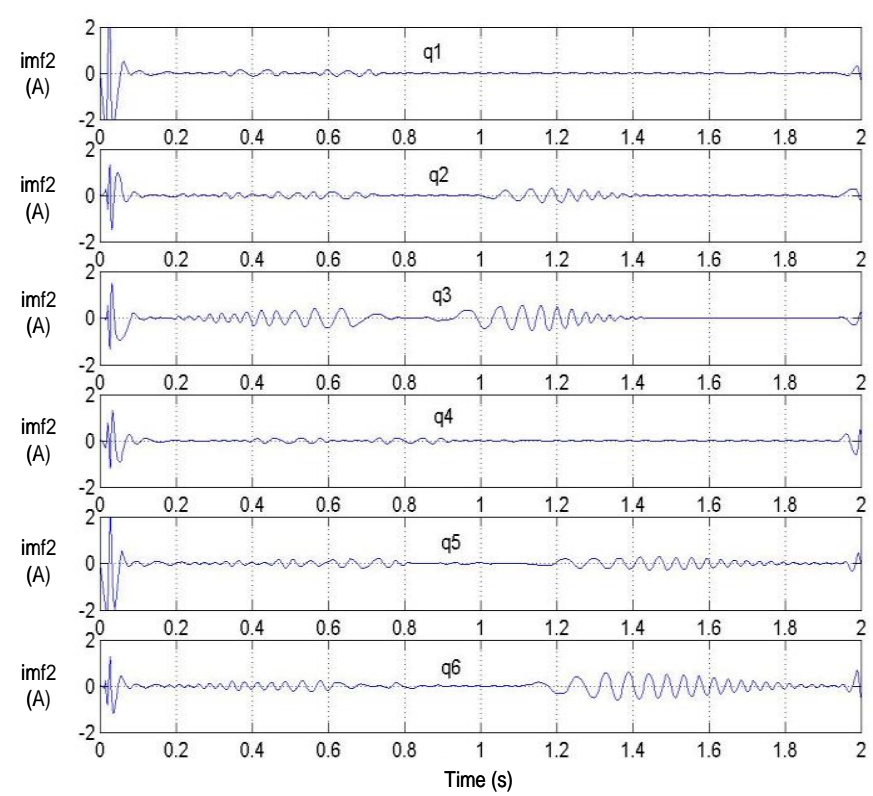

Figure 3. Second intrinsic mode functions (IMF2's) for the six data sets q1 up to q6.
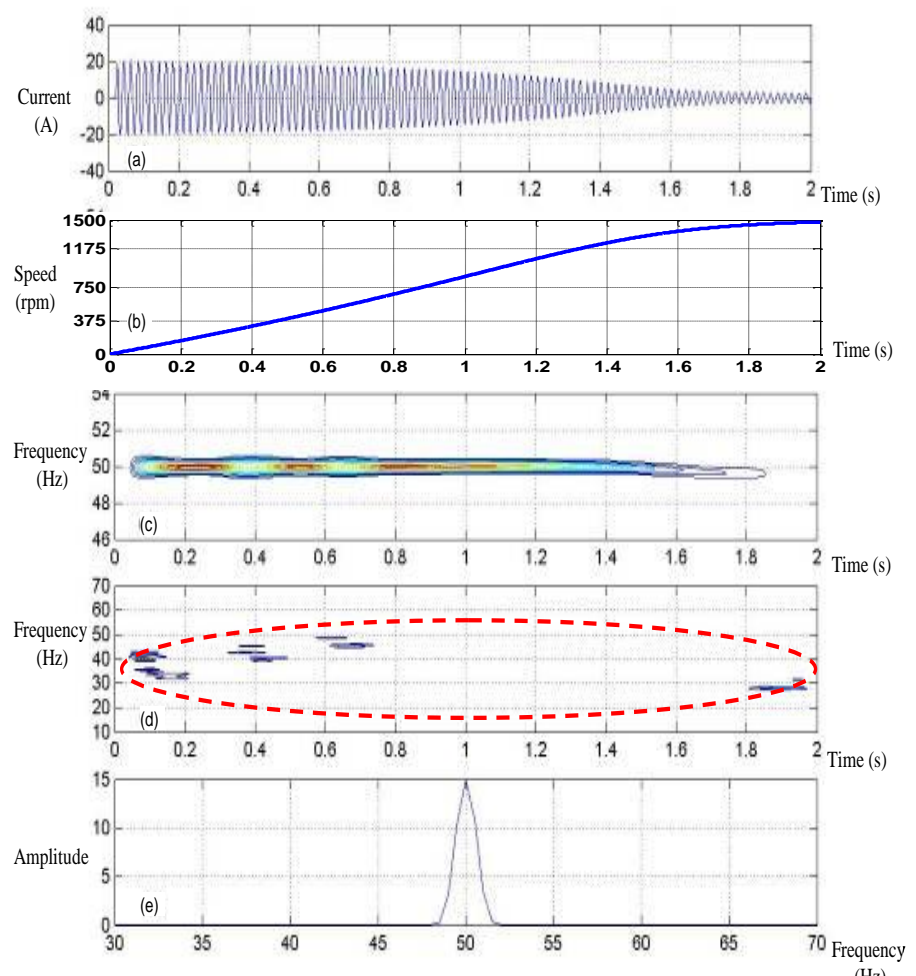

(Hz)

Figure 4. Hilbert-Huang ( $\mathrm{HH}$ ) analysis of the q1-data set: (a) input signal; (b) speed; (c) first intrinsic mode (IMF1) HH spectrum ; (d) second intrinsic mode (IMF2) HH spectrum; (e) input signal power spectral density. 


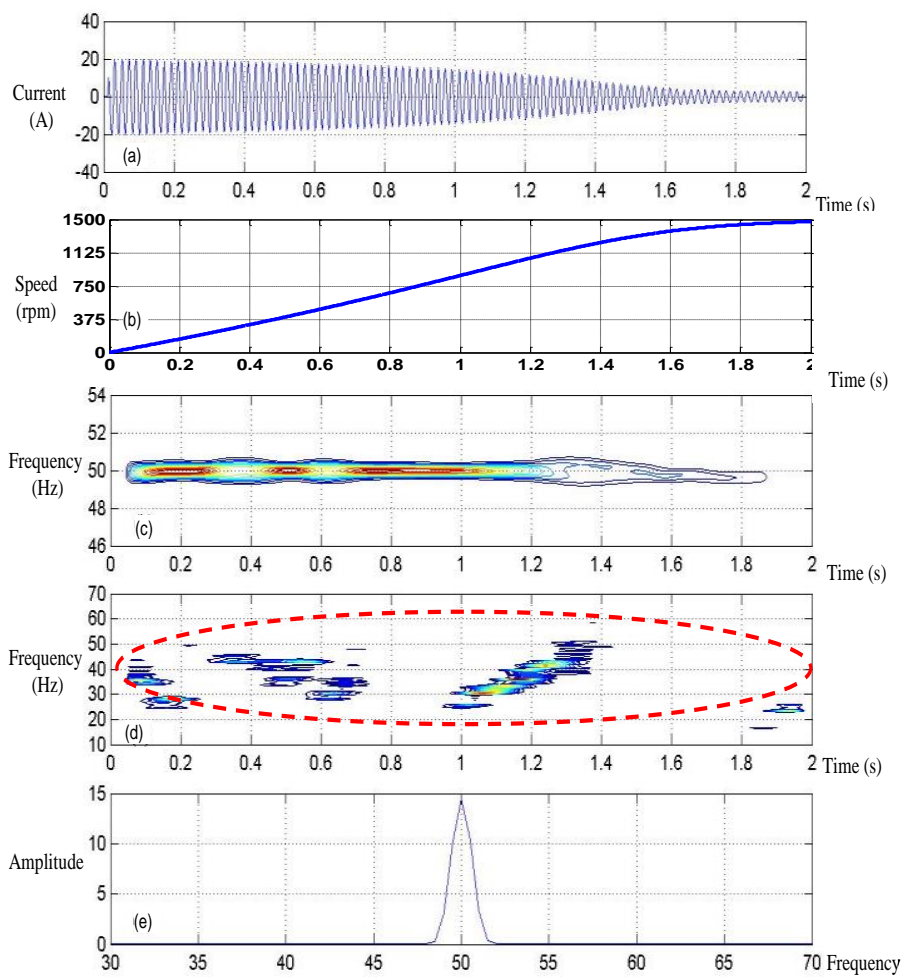

Figure 5. Hilbert-Huang $(\mathrm{HH})$ analysis of the q2-data set: (a) input signal; (b) speed; (c) first intrinsic mode (IMF1) HH spectrum ; (d) second intrinsic mode (IMF2) HH spectrum; (e) input signal power spectral density.

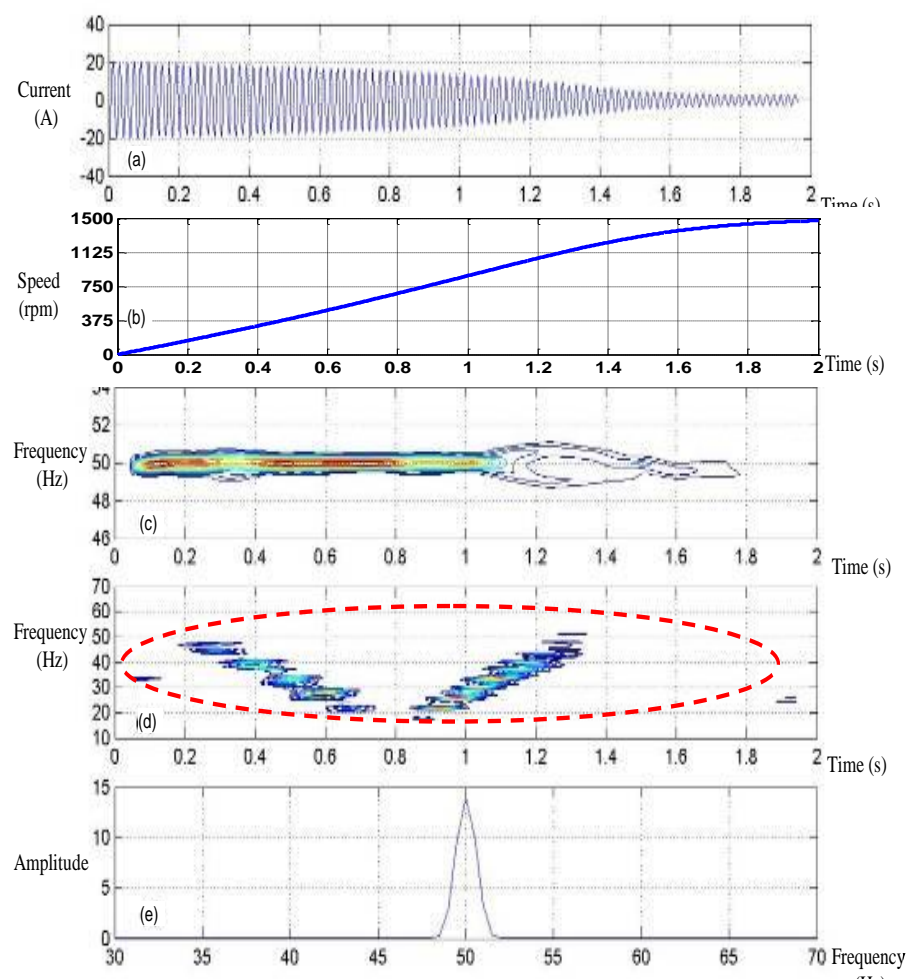

\begin{tabular}{|c|c|}
\hline \multicolumn{1}{c|}{ TABLE I. } & ANALYZED DATA SETS \\
\hline SET & MACHINE CONDITION \\
\hline $\mathrm{q} 1$ & healthy, unloaded \\
$\mathrm{q} 2$ & one broken bar, unloaded \\
$\mathrm{q} 3$ & two broken bars, unloaded \\
$\mathrm{q} 4$ & healthy, loaded \\
$\mathrm{q} 5$ & one broken bar, loaded \\
$\mathrm{q} 6$ & two broken bars, loaded \\
\hline
\end{tabular}

It is interesting to remark how the study of the startup transient enables the detection of the LSH even in the cases of faulty unloaded machines ( $\mathrm{q} 2$ and $\mathrm{q} 3$ ). In this context, the application of the HHT to the startup current shows the presence of the LSH both through the IMF2 and through its $\mathrm{HH}$ spectrum. In these situations of unloaded machine, the application of the classical approach, based on the FFT analysis of the steady-state current would not be suitable for the diagnosis since the slip is very low and the sidebands would almost overlap the supply frequency (a very high frequency resolution would be mandatory for their detection). This can be observed in Fig. 10, in which the FFT spectrum of the steadystate current of the machine with one rotor bar is shown for a slip $s=0.0066$ and for frequency resolution of $0.2 \mathrm{~Hz}$.
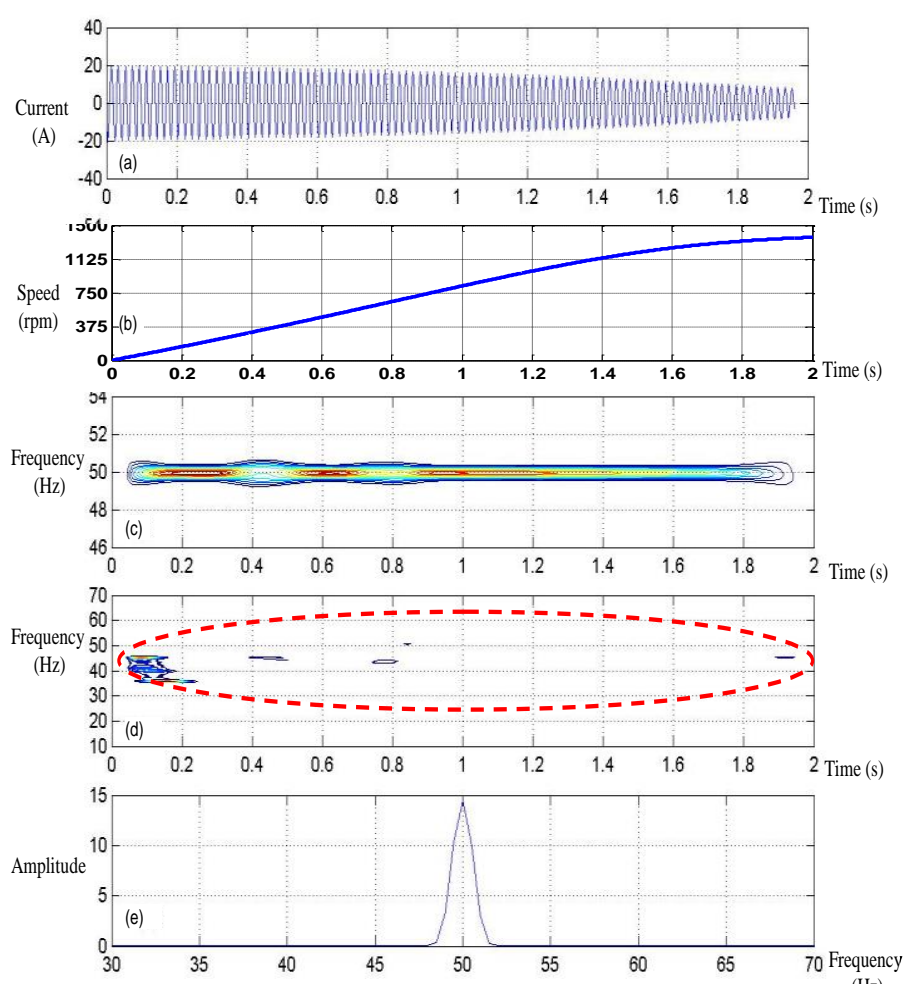

Figure 7. HHT analysis of the q4-data set: (a) input signal; (b) speed; (c) first intrinsic mode (IMF1) HH spectrum; (d) second intrinsic mode (IMF2) HH spectrum; (e) input signal power spectral density.

Figure 6. HHT analysis of the q3-data set: (a) input signal; (b) speed; (c) first intrinsic mode (IMF1) HH spectrum ; (d) second intrinsic mode (IMF2) $\mathrm{HH}$ spectrum; (e) input signal power spectral density. 

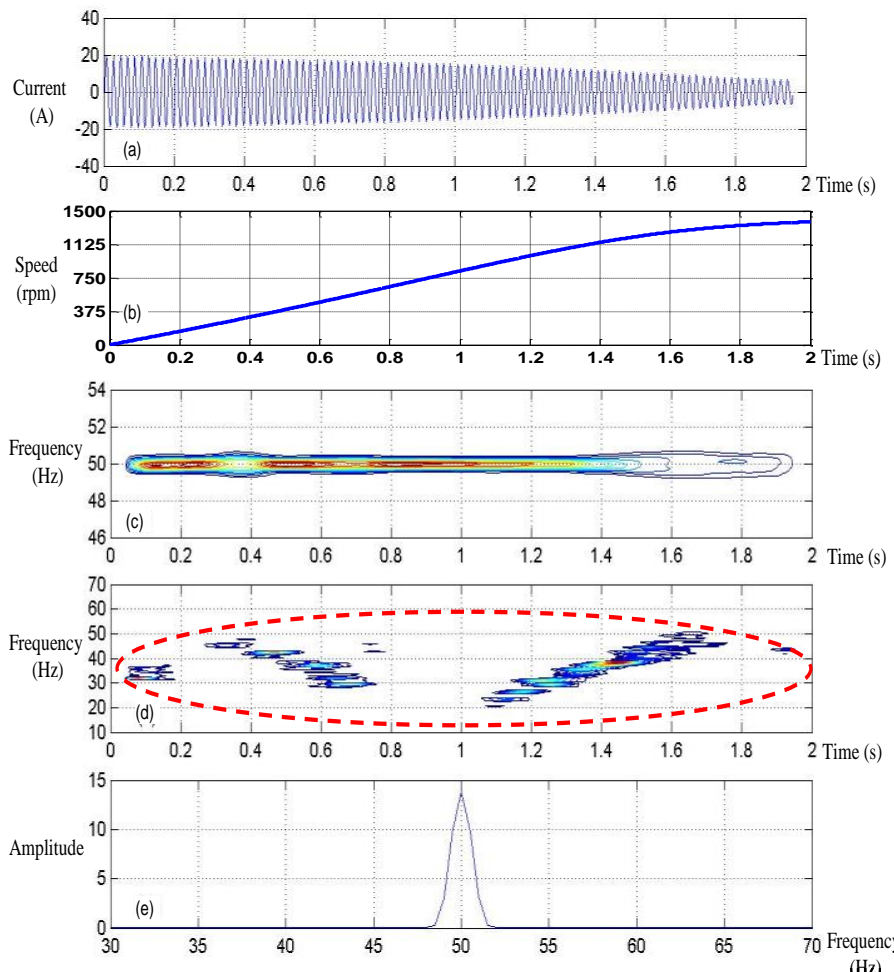

Figure 8 . Hilbert-Huang $(\mathrm{HH})$ analysis of the q5-data set: (a) input signa (b) speed; (c) first intrinsic mode (IMF1) HH spectrum ; (d) second intrinsic mode (IMF2) HH spectrum; (e) input signal power spectral density.
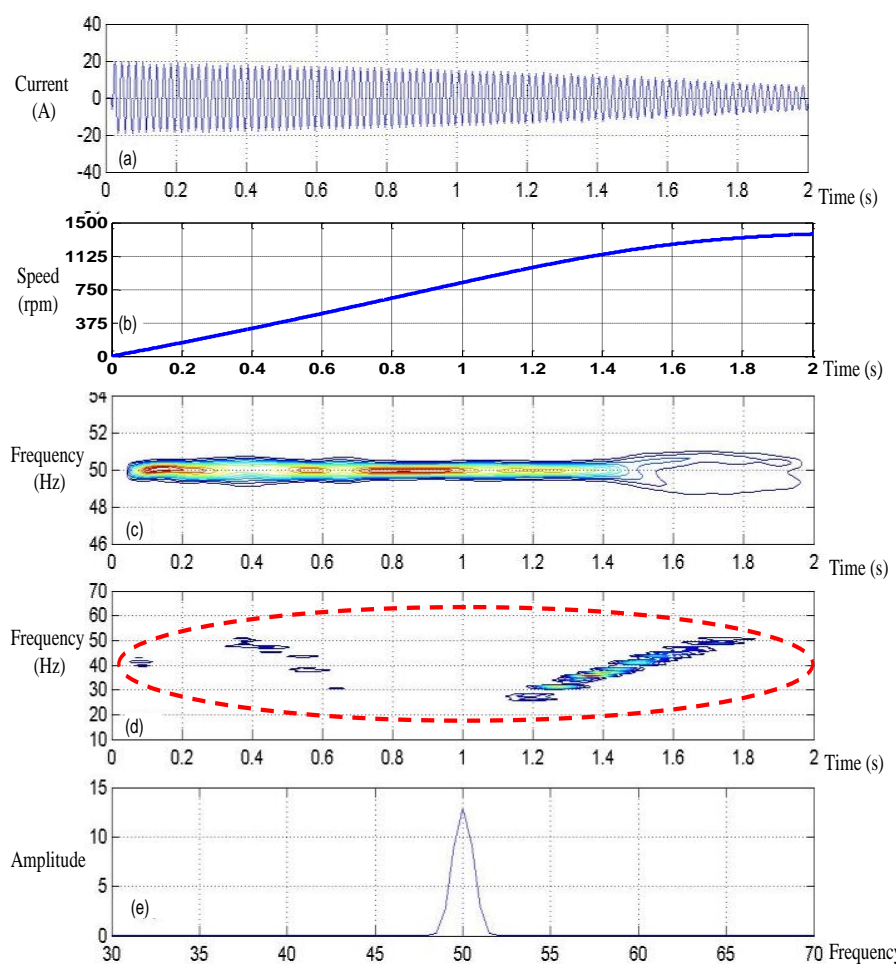

Figure 9. Hilbert-Huang (HH) analysis of the q6-data set: (a) input signal;

(b) speed; (c) first intrinsic mode (IMF1) HH spectrum ; (d) second intrinsic mode (IMF2) HH spectrum; (e) input signal power spectral density.
Moreover, the tool enables to establish a relation between the amplitudes of the IMF2 and the degree of failure in the machine (as it is observed in Fig. 3). This could allow the introduction of non-dimensional parameters for quantifying the degree of severity of the fault, based on the energy of these IMF.

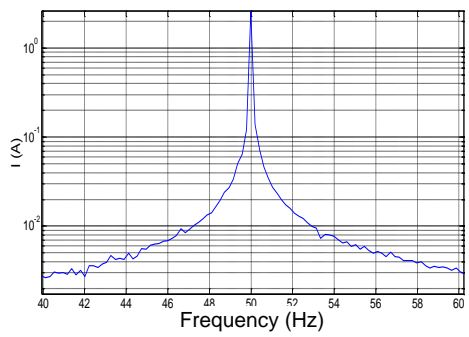

Figure 10. FFT of the steady state current for the unloaded machine with one broken bar.

To sum up, the Hilbert-Huang spectra of the IMF1's do not carry information on the machine status simply providing the electric network supply frequency otherwise easily available from Fourier algorithms. The bulk of information on the machine status is contained in the IMF2 and on its HilbertHuang spectrum as they reveal the presence of the LSH and its time-frequency evolution, these results arising from the empirical signal decomposition and non stationary properties of the Hilbert-Huang algorithm.

\section{B. Application of the DWT Method}

In order to corroborate the results obtained with the HHT, a method proposed in previous works, based on the application of the DWT to the startup current and the subsequent study of the resulting high-order wavelet signals, will be applied. The wavelet signals reflect the evolution of the LSH during the transient. Two alternative approaches were proposed;

- The first is based on the study of the approximation signal with associated frequency band extending up to near the supply frequency. It reflects the evolution in amplitude and frequency of the left sideband during the transient [12].

- The second is based on the study of high-order wavelet signals (one approximation and two detail signals), whose associated frequency bands cover the frequency range between zero and almost the supply frequency. In the case of breakage, the oscillations in those signals follow a characteristic pattern fitting the evolution in frequency of the LSH [7].

DWT was applied to the experimental signals obtained with the $1.1 \mathrm{~kW}$ motors described above. For the DWT analysis, a 6-level decomposition is carried out for the application of the first approach whereas an 8-level decomposition is considered for applying the second one [7]. Daubechines-44 is used as mother wavelet. The frequency bands associated with the wavelet signals used for the analysis are shown in Table II (the sampling rate $f_{s}$ was 5000 samples/sec).

\section{1) Healthy unloaded machine $(s=0.0066)$}

Fig. 11 (a) and (b) show the application of the DWT method to the healthy unloaded machine. As observed in Fig. 
11(a), once the initial oscillations produced by the electromagnetic connection transient are extinguished, the approximation signal $a_{6}$ remains low. This indicates that the LSH is not present, leading to diagnose the healthy condition of the machine. The same conclusion is reached from Fig. 11(b); no oscillations appear in the high-level wavelet signals, after the electromagnetic transient is finished. This is revealing the absence of the LSH and, thus, the healthy condition of the machine.

\section{2) Unloaded machine with 1 broken bar $(s=0.0066)$}

Fig. 12 (a) and (b) show the case of the unloaded machine with one broken rotor bar. Clear differences appear in comparison with Fig. 11. In Fig. 12 (a), clear oscillations occur in the approximation signal $a_{6}$. They fit practically the characteristic evolution in amplitude and frequency of the LSH, commented above.

The reason why they do not fit exactly the theoretical waveform of the LSH (Fig. 1) is that the frequency band corresponding to $a 6$ only extends up to $39.06 \mathrm{~Hz}$. Thus, the evolution of the sideband within the range [39.06 - 50] $\mathrm{Hz}$ is not reflected in that signal. The reason for not setting the upper limit of the frequency band associated with this signal at $50 \mathrm{~Hz}$, is to avoid the interference of the supply frequency component $(50 \mathrm{~Hz})$ which, due to the non-ideal filtering, could be filtered partially in $a 6$, masking the evolution of the LSH.

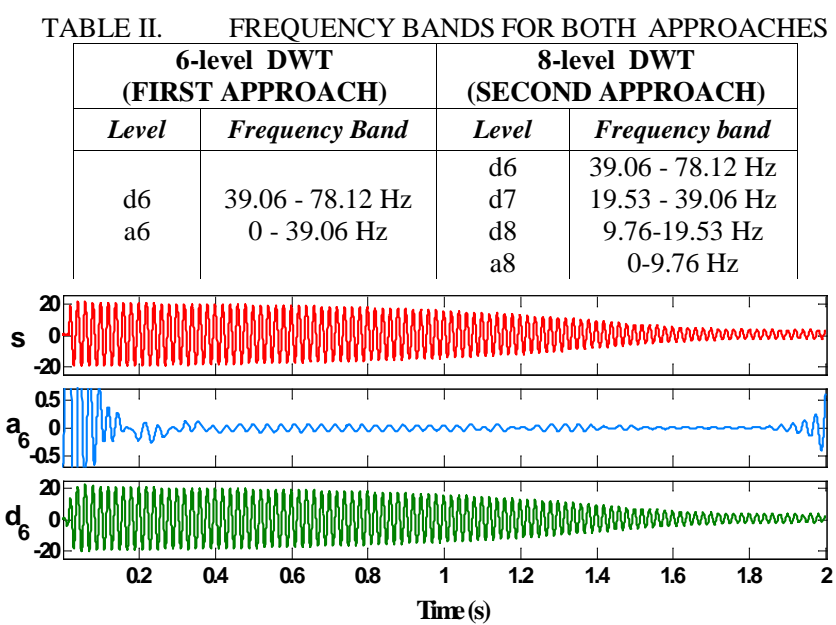

(a)

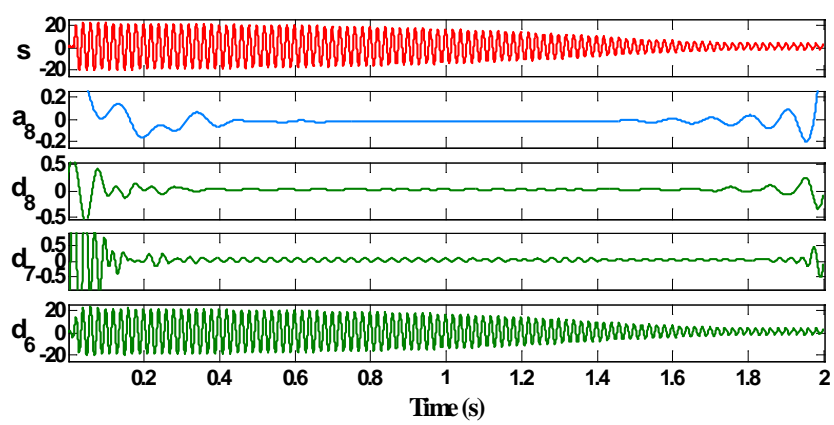

(b)

Figure 11. (a) First approach: 6-level DWT of the startup current for the unloaded healthy machine. (b) Second approach: 8-level DWT of the startup current for the unloaded healthy machine.

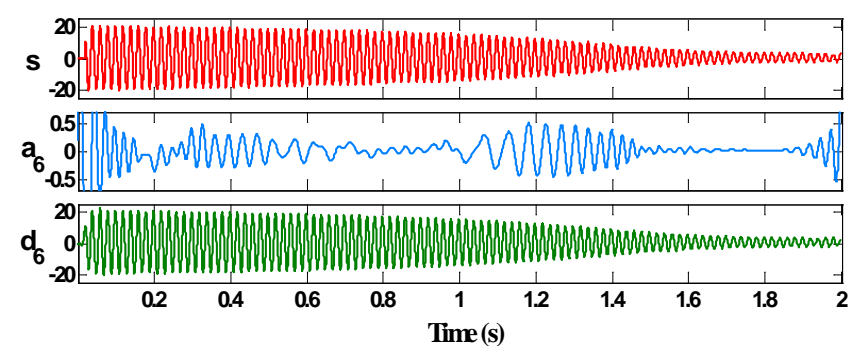

(a)

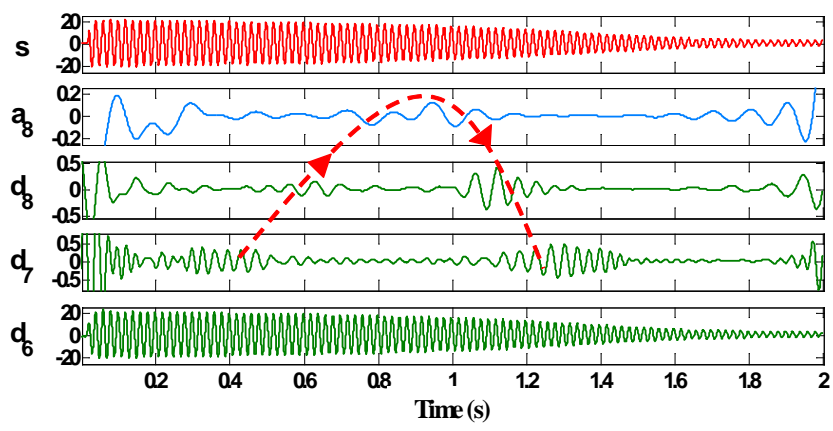

(b)

Figure 12. (a) First approach: 6-level DWT of the current for the unloaded machine with one broken bar. (b) Second approach: 8-level DWT of the current for the unloaded machine with one broken bar.

Application of the DWT method in other cases where the classical Fourier analysis could not lead to a correct diagnosis (pulsating loads, fluctuating supply voltages) can be found in [7]. The method has also been tested in large motors (up to several MW). The results are even clearer than those obtained with small machines and some of them can be found in [26].

An important advantage of the method lies in the fact that it avoids some of the drawbacks of the classical Fourier method, deeply spread in the industrial environment, but maintaining also its simplicity; the only input required is the startup current of a single phase, which can be easy captured in a non-invasive way. Moreover, the method does not require any special software or complex algorithms for the analysis, being suitable any conventional package enabling the DWT of a signal. In addition, the computational requirements are negligible; indeed the number of operations required by the DWT, according to the Mallat algorithm, is much lower than that of the FFT [27].

Despite the excellent results of the method, some issues could be improved. One of them is the selection of the optimum mother wavelet for carrying out the analysis. The experience has shown that this is not a critical matter since different wavelet families (dmeyer, Daubechies, coiflet, symlet, biorthogonal), despite their different mathematical properties, can provide excellent results [13], enabling the reliable extraction of the transient evolution of the left sideband. This is shown in Fig. 13 where the same analysis of Fig 12 (a) is carried out but using dmeyer (an infinite support wavelet) as mother wavelet, instead of Daubechies-44 (compactly supported wavelet). The analogies between both analyses are obvious despite the different characteristics of both mother wavelets. 


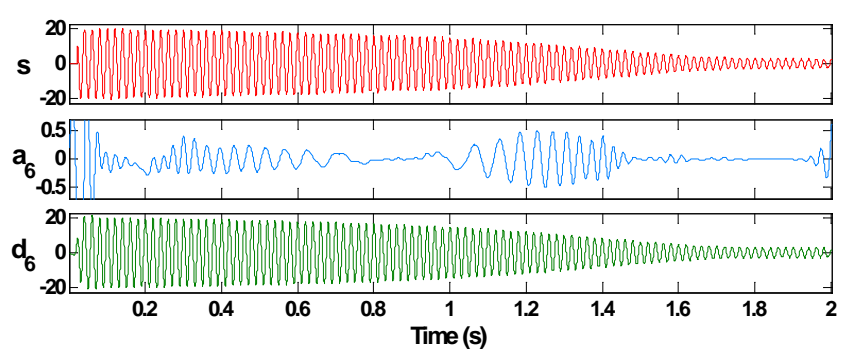

Figure 13. First approach: 6-level DWT of the current for the unloaded machine with one broken bar using dmeyer as mother wavelet.

Nevertheless, when using certain mother wavelets with a low number of coefficients (i.e. a low order Daubechies wavelet), the frequency response of the associated filter becomes worse, increasing the overlap between frequency bands $[19,25]$. This can cause that a portion of the fundamental component is partially filtered into the adjacent band, masking the evolution of the left sideband within the wavelet signal used for the diagnosis. As an example, Fig. 14(a) shows the application of the first approach for the case of the loaded machine with two broken bars, using Daubechies-10 as mother wavelet. Fig. 14(b) shows the same analysis but using Daubechies-44. In the former case, due to the low order of the wavelet, the non-ideal behaviour of the wavelet filter is more visible, leading to a partial filtering of the fundamental component into the adjacent band, which makes not possible the diagnosis. In the second case, when using a high order wavelet, the frequency response improves and the overlap disappears. The same effect appears when using other low order wavelets such as low-order biorthogonal wavelets.

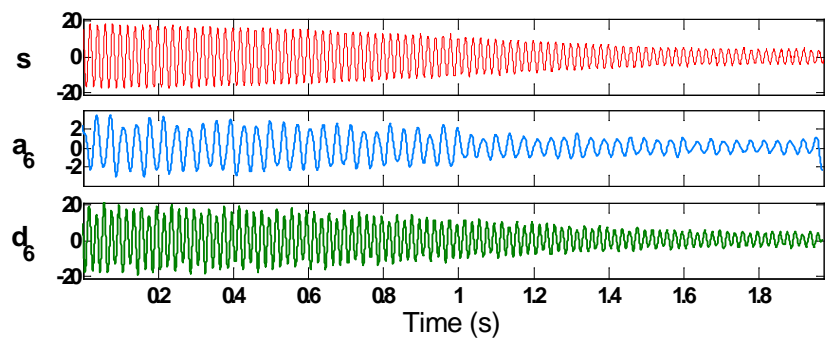

(a)

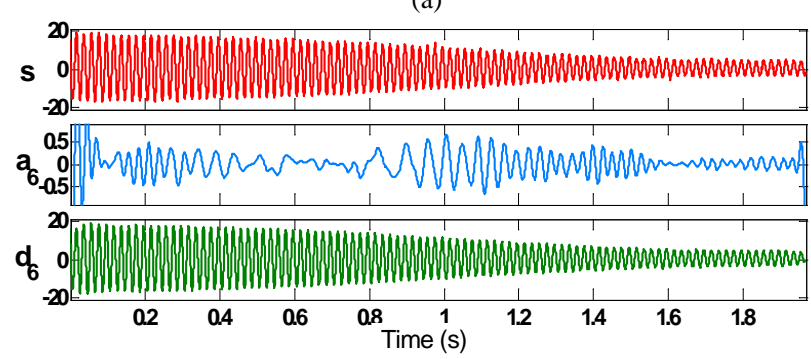

(b)

Figure 14. Application of the first approach for the loaded machine with two broken bars using: (a) Daubechies-10 (b) Daubechies-44.

Another question regarding the DWT is the boundary distortion introduced by the transform. This, together with the initial electromagnetic connection transient, provokes the initial oscillations at the beginning of the wavelet signals observed in
Figs. 3, 4, 6 and 7. This phenomenon might make difficult the identification of the left sideband evolution if the startup is very fast (shorter than half a second) [7]. However, besides the fact that the method works well for small motors like those shown above, it has to be considered that the motors in which the bar breakages are more feasible to occur are large motors started driving high inertias; these are the most favourable conditions for appearing high resistance joints leading to bar breakages [28]. Under these conditions, the startup is much longer than necessary to apply the methodology without any problem. This is reinforced by the fact that these large motors are the ones the diagnosis of which is often most critical since the repair costs in these cases are usually much higher.

\section{Comparison between the methods.}

Both the DWT and the HHT perform a time-frequencyamplitude decomposition of the startup current signal being analyzed. This fact enables the extraction of the time evolution of frequency components associated with the fault, being the most prominent the LSH.

The application of the DWT is simple and the patterns arising from the application of this transform are clear and reliable, becoming a robust tool for the diagnosis of the fault, even in cases where the classical Fourier analysis does not lead to correct results (unloaded machines, fluctuating torque loads, oscillating supply voltage...) [7]. The two proposed DWTbased approaches are complementary; the first one reflects the time evolution of the LSH (in amplitude and frequency) and it fits well its theoretical evolution deduced in previous works; the second approach leads to a characteristic pattern caused by the frequency evolution of the left sideband.

Summarizing the comments made above, possible issues that could be improved regarding the DWT approaches are;

- The mother wavelet selection can constitute a problem if the order of the mother wavelet is low, due to the non-ideal characteristic of the wavelet filtering. This is not a critical problem since a high number of wavelets have been proved as valid for the application of the approaches.

- The boundary effect caused by the transform, together with the initial electromagnetic transient, provoke oscillations at the beginning of the signals, which can distort the patterns used for the diagnosis. This problem is avoided if the startup is long enough since, in this case, the duration of these initial oscillations is negligible. These long startups are common in large motors started under high inertias; In fact, in these motors bar breakages are more likely to occur.

-The dyadic frequency decomposition carried out by the DWT, according to the Mallat algorithm causes that, once the sampling frequency $f_{s}$ is selected, the limits of the bands associated with the wavelet signals are fixed. This can imply a loose of flexibility mainly when studying possible high frequency components introduced by the fault.

- Moreover, due to this fix dyadic decomposition and to the non-ideal filtering of the DWT, the wavelet signals do not extract the whole transient evolution of the LSH (up to the supply frequency), but a substantial part of this evolution (up to near the supply frequency). This does not mean a critical 
problem for the diagnosis, since the characteristic pattern is detected through these signals, but it requires that the possible parameters used for the quantification of the degree of severity of the fault, based on the energy of the wavelet signals [29], should be based on the same sampling rate.

The HHT arises as an alternative tool for the extraction of the transient evolution of the LSH and, so, for the diagnosis of the asymmetry. The intrinsic mode functions (IMF's) and their $\mathrm{HH}$ spectra provide a complementary way for enabling the analysis of the frequency components and their evolution in time and amplitude.

Nevertheless, according to the results obtained here, the patterns do not seem so clear that those obtained with the DWT approaches, perhaps because the $\mathrm{HH}$ spectra are not so easy to be physically interpreted as the wavelet signals. In addition, the use of the HHT also introduces a boundary effect whose influence, as in the DWT, is negligible for long startups. Moreover, there is no an "a priori" relation between the IMF's and the frequency bands, a fact that can create problems for selecting the most suitable number of IMF's to be considered for the detection of the sideband.

In any case, the HHT implies some positive aspects; the dyadic frequency decomposition of the DWT is avoided, due to its operation based on the instantaneous frequency, a fact that could enable a more accurate study of the high frequencies present in the signal. Also the IMF enables a more accurate representation of the theoretical waveform of the LSH, showing the real amplitude of that component, since the frequencies reflected in that IMF signal arrive up to the supply frequency. This might enable the definition of non-dimensional quantification parameters non-dependant on the sampling rate used for capturing the current signal and only based on the energy of the IMF containing the whole evolution of the left sideband. Furthermore, the $\mathrm{HH}$ spectra revealing the timefrequency evolution of the LSH are more suitable to be processed using automatic image recognition techniques. These could enable the faster integration of the tool in portable devices for the on-line diagnosis of the fault.

In conclusion, both approaches can be considered as equivalent tools for the extraction of the same physical phenomenon: the transient evolution of the LSH caused by the rotor asymmetry. Indeed a clear analogy can be established between the approximation signal resulting from the application of the approach 1 based on the DWT and the IMF2 resulting from the application of the HHT method; both of them extract the evolution in amplitude and time of the LSH (Fig. 15 (a)). Equivalently, an analogy can be also made between the high-level wavelet signals resulting from the application of the approach 2 based on the DWT and the HH spectrum of the IMF2; both reflect the characteristic patterns arising due to the evolution in frequency of the LSH during the startup (Fig. 15 (b)).
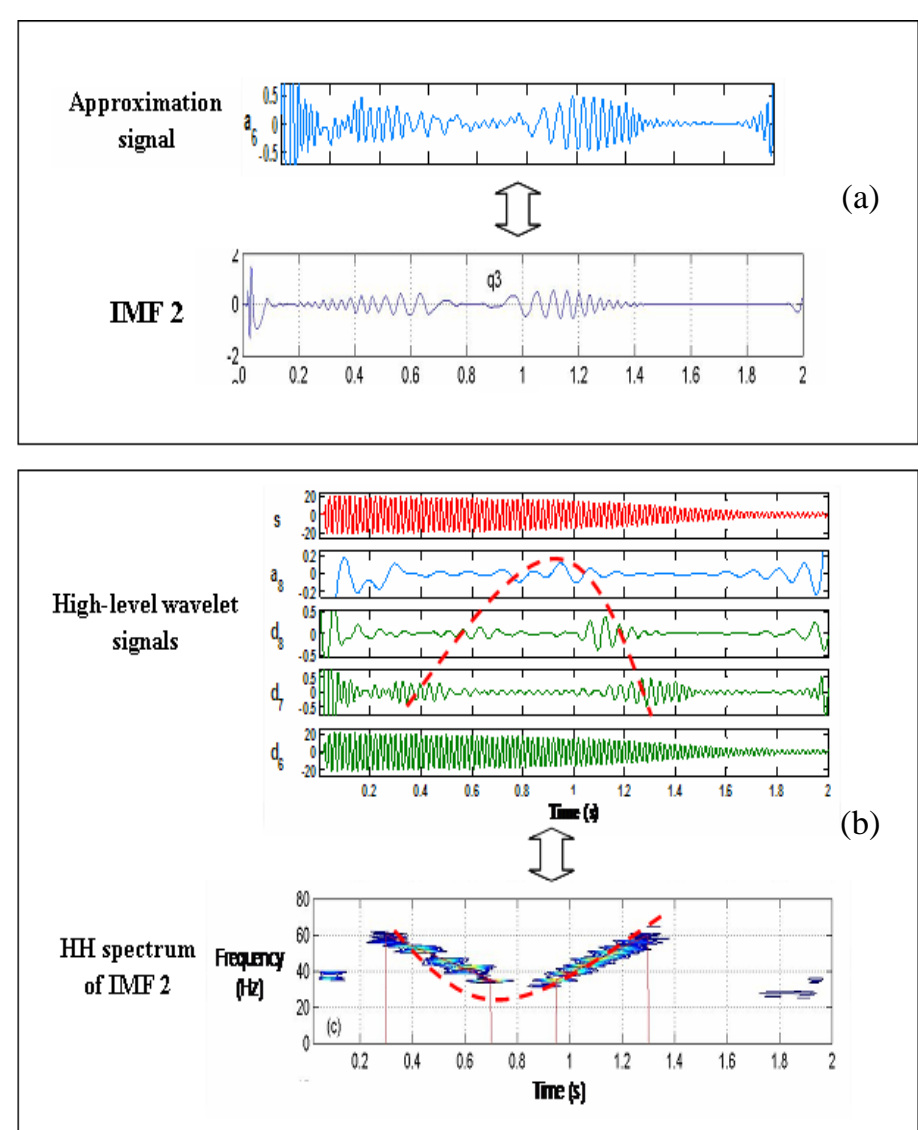

Figure 15. Analogies between: (a) Approximation signal (DWT method) and IMF2 (HHT method); (b) High-level wavelet signals (DWT method) and HH spectrum of IMF2 (HHT method).

\section{CONCLUSIONS}

In this paper the HHT is proposed for the diagnosis of rotor asymmetries in induction machines. Application of the tool on experimental signals is carried out, proving the reliability of the tool even in cases in which the classical FFTbased approach is not suitable (unloaded machines). The results are compared with those obtained using an alternative time-frequency decomposition tool: the DWT.

HHT has some positive aspects in comparison with the DWT such as avoiding the necessity of selection of mother wavelet or a possible better representation of the LSH up to the fundamental frequency, since it avoids the dyadic frequency decomposition of the Mallat algorithm. Also, a better suitability of the $\mathrm{HH}$ spectra for being processed by automatic image recognition algorithms is appreciated. However, the patterns arising from the HHT seem not so clear than with the DWT approaches; in addition, some constraints as the boundary distortion are not avoided and also, the lack of explicit relationships between the IMF's and the frequency of the components reflected by them could make difficult a general formulation of the approach.

In any case, both of them constitute equivalent tools for the extraction of the same physical phenomenon; in this sense, 
important novel analogies are established in the paper between approximation signals and IMF's (amplitude and frequency evolution of the LSH) and between high-level wavelet signals and $\mathrm{HH}$ spectra (characteristic patterns caused by the evolution in frequency).

\section{ACKNOWLEDGEMENTS}

The research leading to these results has received funding from the European Community's Seventh Framework Programme FP7/2007-2013 under Grant Agreement n 224233 (Research Project PRODI "Power plant Robustification based on fault Detection and Isolation algorithms"). The authors also thank 'Vicerrectorado de Investigación, Desarrollo e Innovación of Universidad Politécnica de Valencia' for financing a part of this research through the program 'Programa de Apoyo a la Investigación y Desarrollo (PAID-0607).

\section{REFERENCES}

[1] W.T. Thomson, M. Fenger, "Current signature analysis to detect induction motor faults" IEEE Ind. Appl. Magazine, pp. 26-34, July/August 2001.

[2] D.B.Durocher, G.R. Feldmeier, "Predictive versus preventive maintenance", IEEE Industry Applications Magazine, vol. 10, no.5, pp. 12-21, October 2004.

[3] G.B Kliman, J Stein, and R.D. Endicott, "Noninvasive Detection of Broken Rotor Bars in Operating Induction Motors," IEEE Transactions on Energy Conversion, vol. 3, no. 4, pp. 873-879, December 1988.

[4] R.R. Schoen and T.G. Habetler. "Evaluation and Implementation of a System to Eliminate Arbitrary Load Effects in Current-Based Monitoring of Induction Machines," IEEE Transactions on Industry Applications, vol. 33, no. 6, pp. 1571-1577, November/December 1997.

[5] A. Bellini, F. Filippetti, G. Franceschini, C. Tassoni, and G.B. Kliman, "Quantitative evaluation of induction motor broken bars by means of electrical signature analysis," IEEE Transactions on Industry Applications, vol. 37, no. 5, pp. 1248-1255, September/October 2001.

[6] M.F. Cabanas, M.G. Melero, and G.A. Capolino, "A new methodology for applying the FFT to induction machine on-line diagnosis," in Proceedings of the International Symposium on Diagnostics for Electrical Machines, Power Electronics and Drives (SDEMPED'99), Gijon (Spain), September 1999, pp. 537-543.

[7] J. Antonino-Daviu, M. Riera-Guasp, J. Roger-Folch and M.P. Molina, "Validation of a New Method for the Diagnosis of Rotor bar Failures via Wavelet Transformation in Industrial Induction Machines," IEEE Transactions on Industry Applications., vol. 42, no. 4, pp. 990-996, July/August 2006.

[8] J. Milimonfared, H. Meshgin Kelk, S. Nandi, A. Der Minassians, and H. A. Toliyat, "A novel approach for broken-rotor-bar detection in cage induction motors," IEEE Transactions on Industry Applications, vol. 35, no 5, pp. 1000-1006, September/October 1999.

[9] H. Douglas, P. Pillay, and A. Ziarani , "Broken rotor bar detection in induction machines with transient operating speeds," IEEE Trans. on Energy Conversion, vol. 20, no. 1, pp. 135-141, March 2005.

[10] Z. Zhang and Z. Ren, "A novel detection method of motor broken rotor bars based on wavelet ridge," IEEE Transactions on Energy Conversion, vol. 18, no. 3, pp. 417-423, September 2003.

[11] R. Burnett, J.F. Watson, and S. Elder, "The application of modern signal processing techniques to rotor fault detection and location within three phase induction motors," European Signal Processing Journal, vol. 49, pp. 426-431, 1996.

[12] M. Riera, J. Antonino, J. Roger-Folch and M.P. Molina, "The Use of the Wavelet Approximation Signal as a Tool for the Diagnosis and Quantification of Rotor Bar Failures," IEEE Transactions on Industry Applications, Vol. 44, No. 3, May-June 2008, pp. 716-726.

[13] J. Antonino-Daviu. M. Riera-Guasp, J. Roger-Folch, F. MartínezGiménez and A. Peris "Application and Optimization of the Discrete Wavelet Transform for the Detection of Broken Rotor Bars in Induction
Machines". Applied and Computational Harmonic Analysis, Elsevier, vol. 21, no. 2, pp. 268-279, September 2006.

[14] J. Antonino-Daviu, P. Jover, M. Riera, A. Arkkio and J. Roger-Folch, "DWT Analysis of Numerical and Experimental Data for the Diagnosis of Dynamic Eccentricities in Induction Motors", Mechanical Systems and Signal Processing, Elsevier, vol. 21, no. 6, pp. 2575-2589, August 2007.

[15] F. Briz, M. Degner, P. Garcia, D. Bragado, "Broken Rotor Bar Detection in Line-Fed Induction Machines Using Complex Wavelet Analysis of Startup Transients," IEEE Transactions on Industry Applications, Vol. 44, No. 3, May-June 2008, pp. 760-768.

[16] N.E. Huang, S.S.P Shen, Hilbert-Huang Transform and its Applications. World Scientific Publishing, 2005

[17] D. Yu, J. Cheng and Y. Yang, "Application of EMD method and Hilbert spectrum to the fault diagnosis of roller bearings", Mechanical Systems and Signal Processing, Elsevier, Vol. 19, 2005, pp. 258-270.

[18] V.K. Rai, A.R. Mohanty, "Bearing Fault Diagnosis using FFT of intrinsic mode functions in Hilbert-Huang transform," Mechanical Systems and Signal Processing, Elsevier, Vol. 21, No. 6, August 2007, pp. 26072615.

[19] A.P. Bradley and W.J. Wilson, "On wavelet Analysis of Auditory evoked Potentials," Clinical neurophysiology, Elsevier, vol. 115, no. 5, pp. 1114-1128, 2004.

[20] R. Polikar, L. Udpa, S.S. Udpa, T. Taylor, "Frequency Invariant Classification of Weld Inspection Signals", IEEE Tran. Ultra. Ferro and Freq. Cont., vol. 45, no. 3, pp.614-625, 1998.

[21] W. Deleroi, "Squirrel cage motor with broken bar in the rotor - Physical phenomena and their experimental assessment," in Proc. of ICEM'82. Budapest, Hungary, 1982, pp. 767-770.

[22] N.E. Huang, Z. Shen, S.R. Long, M.C. Wu, H.H. Shih, Q.Zheng N.C. Yen,C.C. Tung and H.H. Liu, "The Empirical Mode Decomposition and the Hilbert Spectrum for Nonlinear and Nonstationary Time Series Analysis", Proc. Royal Society of London, A, vol. 454, pp. 903-995, 1998

[23] D. Gabor, "Theory of Communication," J. IEE, vol. 93, no. 26, pp. 429457, November 1946

[24] Z.K. Peng, P.W. Tse, F.L. Chu, "A Comparison Study of Improved Hilbert-Huang Transform and Wavelet Transform: Application to Fault Diagnosis for Rolling Bearing", Mechanical Systems and Signal Processing, Elsevier, Vol. 19, 2005, pp. 974-988.

[25] T. Tarasiuk, "Hybrid wavelet-Fourier Spectrum Analysis," IEEE Transactions on Power Delivery, vol. 19, no. 3, pp. 957-964, July 2004.

[26] J. Antonino, J. Rusek, M. Riera and J. Roger-Folch, "Low Frequency Filtering for the Diagnosis of Cage Asymmetries in Induction Machines", in Proc. XVII International Conference on Electrical Machines ICEM 2006, Crete, Greece, September 2-5, 2006 (CD-ROM).

[27] C.S. Burrus, R.A. Gopinath and H. Guo, Introduction to Wavelets and Wavelet Transforms. A primer, Prentice Hall, 1998.

[28] J.M. Cubert, "Use of electronic controllers in order to increase the service life on asynchronous motors", Proc. European seminar on electro-technologies for industry, Bilbao, May 1992, pp.393-404.

[29] M. Riera, J. A. Antonino, J. Roger-Folch, and M.P. Molina, "Definition of DWT-based parameters for the quantification of rotor bar breakages in industrial induction machines", in Proc. XII International Symposium on Electromagnetic Fields in Mechatronics, Electrical and Electronic Engineering, ISEF 2005, Baiona, Spain, September 17-19, 2005

[30] R. Wieser, C. Kral, F. Pirker, M. Schagginger, "Sequences of FieldOriented Control for the Detection of Faulty Rotor Bars in Induction Machines-The Vienna Monitoring Method", IEEE Transactions on Industrial Electronics, vol. 47, no. 5, pp. 1042-1050, October 2000. 


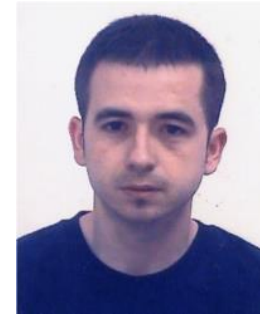

Jose A. Antonino-Daviu received the M.Sc. degree in Electrical Engineering from the Universidad Politecnica de Valencia in 2000 and the Ph.D. degree in Electrical Engineering in 2006. He worked in the private sector, being involved in several international projects. Currently, he is Associate Professor in the School of Industrial Engineering of the mentioned University, where he develops his docent and research work. His primary research interests are condition monitoring of electric machines, wavelet theory and its application to fault diagnosis and design and optimization of electrical installations and systems.

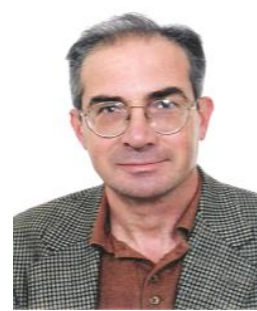

M. Riera-Guasp received the M.Sc. degree in Industrial Engineering and the Ph.D. degree in Electrical Engineering from the Polytechnic University of Valencia (Spain) in 1981 and 1987, respectively. Currently he is an Associate Professor in the Department of Electrical Engineering of the Polytechnic University of Valencia. His research interests include condition monitoring of electrical machines, applications of the Wavelet Theory to electrical engineering and efficiency in electric power applications.

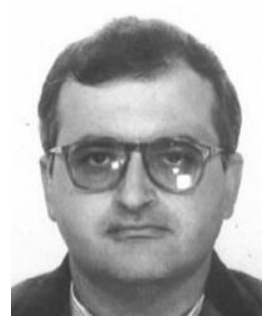

M. Pineda-Sanchez was born in 1962 in Albacete (Spain). He received his Dipl. Ing. and Dr. Ing. degrees in electrical engineering from the Universidad Politécnica de Valencia (Spain) in 1985 and 2004, respectively. $\mathrm{He}$ joined the faculty of the Universidad Politécnica de Valencia in 1987 as professor in the Department of Electrical Engineering, in the area of theory and control of electrical machines. His research interests include electrical machines and drives, induction motor diagnostics, numerical simulation of electromagnetic fields, and software development.

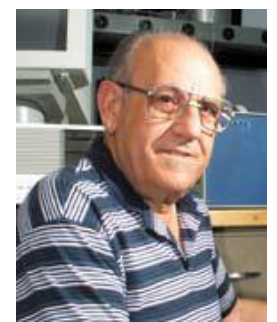

Rafael B. Pérez is Master in Chemistry from University of Valencia (1951), Master in Nuclear Engineering from MIT (Massachusets Institute of Technology) (1958) and $\mathrm{PhD}$ in Physics from University of Madrid (1961).

$\mathrm{He}$ has worked for: Spanish Air Force Meteorology Officer (1948-1955); Junta de Energia Nuclear (Spain) (1955-1961); Associate Professor of Nuclear Engineering University of Florida (1961-1967); Senior Research Scientist Oak Ridge National Laboratory (ORNL) (1967-1980), Full Professor of Nuclear Engineering University of Tennessee (UTK) (1980 - 1997).

Presently, he is Emeritus Professor of UTK He has over 200 reviewed papers on signal analysis, nuclear reactor dynamics, quantum mechanics and neutron cross section theory.

He is Fellow of the American Nuclear Society (ANS); in 1999 he was awarded with the ANS Wigner Prize. 